\title{
Level of nutrition knowledge and its association with fat consumption among college students
}

\author{
Najat Yahia ${ }^{1 *}$, Carrie A. Brown ${ }^{2,4}$, Melyssa Rapley ${ }^{1}$ and Mei Chung ${ }^{3}$
}

\begin{abstract}
Background: Intake of saturated fat, trans fat, and cholesterol has been associated with increased risk of coronary heart disease. The aim of this study was to explore whether increased nutrition knowledge is associated with a reduction in the consumption of unhealthy fats in a sample of university students.

Methods: A sample of 231 students, with a mean age of 20 years, was recruited from university campus during spring 2012. Students completed a validated questionnaire related to students' demographic, nutrition knowledge, and daily fat consumption. Weight, height, and waist circumference were measured. Data were analyzed using oneway ANOVA, chi-square, and student's t-test.

Results: Results indicate that female students have greater nutrition knowledge than male students (the mean nutrition score for women was 5 points higher than that of men $(P=0.01)$ ). Nutrition knowledge was negatively correlated with fat and cholesterol intake. Students who consumed more than $35 \%$ calories from fat or $>300 \mathrm{mg}$ of cholesterol daily had lower mean nutrition scores than those students with lower fat or cholesterol intake (8 points lower and 7.9 points lower, respectively). Using linear regression for nutrition scores on estimated saturated fat intake and cholesterol intake (controlling for gender, height, weight, age, and dieting), nutrition scores were negatively associated with saturated fat intake $(-0.15, P<0.0001)$ and cholesterol intake $(-1.38, P<0.0001)$.

Conclusion: Students with greater nutritional knowledge consumed less unhealthy fats and cholesterol. This finding magnifies the role of nutrition education as a potential tool in health campaigns to promote healthy eating patterns among college students. Results of this pilot study can inform the design of future nutrition education intervention studies to assess the efficacy of nutrition knowledge on pattern of fat consumption among college students.
\end{abstract}

Keywords: Nutrition education, Fat consumption, Nutritional knowledge, University students

\section{Background}

Cardiovascular disease (CVD) is the leading cause of death in the United States $[1,2]$. According to the Centers for Disease Control and Prevention (CDC), about 600,000 American adults die annually of heart disease, or 1 of every 4 deaths [3]. According to a report published by the Michigan Department of Community Health in 2015, the number of cases of heart disease in Michigan is projected to rise from 600,000 to 2.9 million

\footnotetext{
*Correspondence: yahia1n@cmich.edu

'Department of Human Environmental Studies, Central Michigan University, Wightman 108, Mt. Pleasant, MI 48859, USA

Full list of author information is available at the end of the article
}

by year 2030 [4]. Recent estimates from the 2013 Behavioral Risk Factor Surveillance System (BRFSS) indicated that $40.6 \%$ of Michigan adults aged 18 years and older reported having high blood cholesterol levels, with higher prevalence among white non-Hispanic adults (42.2 \%) than Black, non-Hispanic adults (36.1\%) [5].

A diet high in saturated fat, trans fat, and cholesterol is known to raise levels of serum blood cholesterol and can negatively impact cardiovascular health [6]. Accordingly, the American Heart Association recommends that healthy adults should limit their intake of saturated fat to less than $7 \%$ of total daily calories, trans fat to less than $1 \%$ of total daily calories, and cholesterol to less 
than $300 \mathrm{mg}$ a day [7, 8]. Likewise, the 2010 Dietary Guidelines for Americans call for reductions in the consumption of saturated and trans fat such as cream, butter, beef fat (tallow, suet), chicken fat, pork fat (lard), stick margarine, and shortening [9].

College is a critical period in life since many lifestyle habits are formed and may persist into adulthood, thereby impacting health [10-13]. Several investigators have reported unhealthy dietary practices among college students such as increasing consumption of fast food and high-fat foods among college students [9-21]. Butler et al. reported significant increases in the percentage of fat and the number of alcoholic beverages consumed daily in a sample of female students at a Midwestern university in the U.S. during their first year of college [20]. Other authors reported a significantly higher intake of total and saturated fat and a lower intake of polyunsaturated and monounsaturated fat in a sample of university students, when compared to the American Heart Association recommendations [21]. The U.S. Surgeon General, in two previous reports, titled "Nutritional Prevention Strategy/Health Eating" and "The Surgeon General's Vision for a Healthy and Fit Nation", identified universities and schools as sites to raise awareness and educate students about nutrition to help them understand and apply the Dietary Guidelines for Americans recommendations to reduce their risk for heart disease $[22,23]$.

Literature on the impact of nutrition education on dietary practices has been mixed. Several investigators have reported that nutrition education may change students' dietary habits and food choices [24-28] while others reported no significant correlation between nutrition knowledge and food choices [29, 30]. Indeed, there could be many factors that impinge on a students' dietary behaviors, but some basic understanding of nutrition is necessary for a diet change to occur [31]. Whether nutrition education can provide students with the power to select healthier food choices and how well students apply food-related knowledge into their daily dietary practices deserve further attention. Thus, this pilot study aimed to explore whether higher nutrition knowledge level is associated with a lower level of fat consumption in a sample of university students from Central Michigan University.

\section{Methods}

\section{Design and sample}

This study was a cross-sectional survey. A sample of 231 students (71 \% females and $29 \%$ males), with a mean age of 20 years, was drawn from the university campus. Students were recruited in the classroom (Foods and Nutrition classes) and online (Blackboard announcements) by a Central Michigan University (CMU)
Nutrition and Dietetics professor during fall 2011spring 2012 semesters. The Food and Nutrition classes were introductory nutrition classes offered to all students from any majors and are considered as University Program (UP) - General Education courses. Students who enroll in these classes have no prior academic nutrition knowledge since these classes are introductory nutrition classes offered to all undergraduate students from any majors as part of UP/General Education courses irrespective of students' year in school. Students agreeing to participate were asked to sign a consent form, in harmony with the Helsinki declaration, and to come to a laboratory classroom for anthropometric measurements and to receive a numerical code for completing a self-administered online questionnaire that included questions related to students' demographic, nutrition knowledge, and daily fat consumption. The questionnaire was available online via SurveyMonkey Pro (SurveyMonkey.com, LLC, Palo Alto, CA) for about 10 weeks to accommodate students' response times. Students were not offered any incentives for their participation and were informed that they could withdraw from the study at any time.

Two hundred and fifty students participated in this study, however 19 students were excluded as they did not complete the entire questionnaire. The study protocol was approved by the CMU Institutional Review Board (IRB).

\section{Data collection}

Data were collected in two steps. First, anthropometric parameters, including weight, height, percentage body fat, visceral fat score and waist circumference, were measured by a CMU Nutrition and Dietetics professor.

Weight, percentage body fat, visceral fat, and body mass index were measured using a Tanita bioelectrical impedance analyzer (BIA) SC-331S (Tanita Corporation, Chicago, USA). For BIA measurements, the student's height, age, and gender were entered into the machine before testing. Then, the student stepped into the BIA's footpads with bare feet (both feet touched the electrodes). Weight, percentage body fat, visceral fat, and body mass index were recorded from the BIA readings. Since severe dehydration or over-hydration can affect BIA readings, participants' measurements were taken in the morning after overnight fasting and without consuming alcohol or any stimulant, on an empty bladder, and with no intense exercise. Height was measured while a student was standing erect and without shoes using a stadiometer (Seca 217 Height Measuring Stadiometer, Quick Medical, Issaquah, WA, USA).

Body mass index (BMI) was used to assess students' body weight category. According to the BMI guidelines published by the National Institutes of Health, participants were 
categorized into four groups as follows: underweight (BMI $\leq 18.5)$, normal weight $(18.5 \leq \mathrm{BMI}<24.9)$, overweight $(25 \leq \mathrm{BMI}<30)$, and obese $(\mathrm{BMI} \geq 30)$ [32]. The healthy range for body fat percentage was considered as 8-19\% for males and 17-32\% for females (Tanita BIA SC-331S body fat ranges for healthy adults). A visceral fat rating from 1 to 12 was considered healthy for all, and 13 - 59 indicated an excess level of visceral fat. Waist circumference was recorded with a flexible, non-stretchable measuring tape according to the National Institutes of Health guidelines [32].

\section{Online questionnaire}

In the second step, students were asked to complete an online questionnaire administered via SurveyMonkey (https:// www.surveymonkey.com). Students were told that they could withdraw from the survey at any time and were given an option to skip questions. The questionnaire was pilot-tested on 20 students before it was administered to the students in this study. It consisted of questions related to students' demographics, dietary fat intake, and nutritional knowledge, and was divided into 3 main parts as follows:

Part I - Demographics: 8 questions related to age, gender, major of study (Science (such as Nutrition and Dietetics, Health and Fitness, Exercise Science,

Kinesiology, Community Health) or non-Science major (such as Music, Religion, Theater, Philosophy), year in school, ethnicity, place of residence (on-campus/offcampus), dieting, and smoking status.

Part II - Block Dietary Fat Screener: a validated 17item food frequency questionnaire (FFQ) was used to assess students' usual fat intake (Block Fat Screener, NutritionQuest, Berkeley, CA, USA) [33]. A description of the questionnaire is presented in reference 33. The fat screener includes questions about commonly consumed high-fat foods (41 food items) and designed to rank individuals with regard to their usual total fat intake. Data collected from the fat screener were analyzed using prediction equations to generate point estimates of total fat (grams), saturated fat (grams), percent calories from fat, and cholesterol (mg).

Part III - Nutrition Knowledge: 50 questions related to nutritional knowledge. This part was taken from a previously published study by Parmenter and Wardle [34], whose approval was obtained prior to the study. This part includes four sections (dietary recommendations, sources of nutrients, everyday food choices, and diet-disease relationship), each section assessing a different aspect of general nutrition knowledge as follows: section 1- knowledge of recommendations regarding increasing and decreasing intake of different food groups; section 2- nutrient knowledge; section 3- food choice; and the last section (section 4) about the relationships between diet and disease. The questions were in the form of multiple choice, fill-in-the-blank, and check mark. Each question carries one point for a correct answer. Section 1 (dietary recommendations) has a maximum score of 11; Section 2 (sources of foods/ nutrients) has a maximum score of 69; Section 3 (choosing everyday foods) has a maximum score of 10; Section 4 (diet-disease relationships) has a maximum score of 20. Students' responses to these sections were scored according to the nutrition knowledge questionnaire's scoring scale [34], and the scores were summed to obtain the nutrition knowledge score. A detailed description of the nutrition knowledge questionnaire and the scoring scale are available at: https://www.ucl.ac.uk/hbrc/resources/resources_eb and described elsewhere [34].

\section{Data analysis}

Power calculation was not performed because this study is exploratory in nature to inform the design of future studies. Statistical analyses were performed using the SAS (9.3, U.S.A) software. Students' results on the $t$-test and Chi-square test for independence were used to examine differences in the anthropometric characteristics between male and female students. Results are expressed as means \pm SD (standard deviation). One-way ANOVA was used to test differences in fat, saturated fat, and cholesterol intake for various demographic variables. Student's $t$-test results were used to examine differences in nutrition score by gender, high fat consumption ( $>35 \%$ calories), and high cholesterol ( $>300 \mathrm{mg} /$ day). Separate linear regression for nutrition scores on estimated saturated fat intake and cholesterol intake (controlling for gender, height, weight, age, and self-reported dieting), were used to analyze the association between nutrition scores and saturated fat or cholesterol consumption. Partial correlation coefficients (controlling for gender, height, weight, and age) between nutrition scores and saturated fat intake and cholesterol intake were also calculated. All reported $p$ values were based on 2 -sided tests with a significance level of $5 \%$ (Additional files 1, 2, 3, 4 and 5.

\section{Results}

\section{Participants' characteristics}

Two hundred and thirty one students $(71 \%$ females and $29 \%$ males), with a mean age of $20.6 \pm 2.0$ years, participated in this study. The average weight was $69.8 \pm$ $16.7 \mathrm{~kg}$, with an average height of $166.8 \pm 9.1 \mathrm{~cm}$. The mean BMI was $24.2 \pm 4.4 \mathrm{~kg} / \mathrm{m}^{2}$. As for percentage body fat, the mean value was $23.9 \pm 8.6 \%$. The mean values of visceral fat scores for males and females were $1.9 \pm 1.8$ and $3.7 \pm 3.4$, respectively (Table 1 ). Based on BMI, the majority of students $(68 \%)$ were within the healthy 
Table 1 Students' characteristics

\begin{tabular}{|c|c|c|c|c|}
\hline & Male $N=67$ & Female $N=164$ & Total $N=231$ & $P$ Value \\
\hline Age & $21.3 \pm 2.4$ & $20.3 \pm 1.6$ & $20.6 \pm 2.0$ & 0.0002 \\
\hline Weight & $83.9 \pm 16.9$ & $64.0 \pm 12.7$ & $69.8 \pm 16.7$ & $<.0001$ \\
\hline Height & $176.7 \pm 6.6$ & $162.9 \pm 6.5$ & $166.8 \pm 9.1$ & $<.0001$ \\
\hline Waist circumference & $91.1 \pm 11.1$ & $81.1 \pm 9.6$ & $83.6 \pm 11.0$ & $<.0001$ \\
\hline BMI & $26.0 \pm 4.9$ & $23.4 \pm 4.0$ & $24.2 \pm 4.4$ & $<.0001$ \\
\hline$\%$ body fat & $16.7 \pm 8.1$ & $26.8 \pm 6.9$ & $23.9 \pm 8.6$ & $<.0001$ \\
\hline Visceral fat score & $3.7 \pm 4.2$ & $1.9 \pm 1.8$ & $2.4 \pm 2.8$ & $<.0001$ \\
\hline Ethnicity & & & & 0.0905 \\
\hline White & $85 \%$ & $92 \%$ & $90 \%$ & \\
\hline Black/other & $15 \%$ & $8 \%$ & $10 \%$ & \\
\hline Body mass index & & & & 0.0003 \\
\hline $\mathrm{BMI}<18.5$ & $3 \%$ & $1.7 \%$ & $2.6 \%$ & \\
\hline BMI 18.5- 24.9 & $47.8 \%$ & $76.2 \%$ & $68.0 \%$ & \\
\hline BMI > 25 (overweight) & $35.8 \%$ & $17.1 \%$ & $22.5 \%$ & \\
\hline BMI > 30 (obese) & $13.4 \%$ & $4.3 \%$ & $6.9 \%$ & \\
\hline Academic level of study & & & & 0.6660 \\
\hline First year & $14 \%$ & $13 \%$ & $14 \%$ & \\
\hline Second year & $20 \%$ & $27 \%$ & $25 \%$ & \\
\hline Third year & $26 \%$ & $27 \%$ & $27 \%$ & \\
\hline Fourth year & $20 \%$ & $19 \%$ & $19 \%$ & \\
\hline Fifth year & $20 \%$ & $13 \%$ & $15 \%$ & \\
\hline \multicolumn{5}{|l|}{ Major of study } \\
\hline Health science & $37 \%$ & $56 \%$ & $51 \%$ & 0.0078 \\
\hline Non- health science & $63 \%$ & $44 \%$ & $49 \%$ & \\
\hline Current place of residence & & & & 0.6493 \\
\hline On-campus & $30.0 \%$ & $32.9 \%$ & $32.0 \%$ & \\
\hline Off-campus & $70.0 \%$ & $67.1 \%$ & $68.0 \%$ & \\
\hline \multicolumn{5}{|l|}{ Currently dieting } \\
\hline No & $80.6 \%$ & $82.3 \%$ & $81.8 \%$ & 0.7584 \\
\hline Yes & $19.4 \%$ & $17.7 \%$ & $18.18 \%$ & \\
\hline \multicolumn{5}{|l|}{ Smoking } \\
\hline Non-smokers & $81 \%$ & $93 \%$ & $90 \%$ & 0.0163 \\
\hline Smokers & $11 \%$ & $4 \%$ & $5 \%$ & \\
\hline Former smokers & $9 \%$ & $3 \%$ & $5 \%$ & \\
\hline
\end{tabular}

weight category, particularly female students, and about one-third were either overweight (22.5\%) or obese (6.9\%). Of the participating students, $90 \%$ reported as white, and $10 \%$ were African American or other ethnic origin, reflecting the composition of ethnic groups at CMU. Fifty-one percent of students were science majors with a higher percentage for females than males (56\% vs. $37 \%, P=0.0088)$. More than two-thirds of the students $(70 \%)$ lived outside of campus, and $80.9 \%$ reported not following any special diet. The percentages of 2nd and 3rd year students were $25 \%$ and $27 \%$, respectively, while the other 3 levels (1st, 4th, and 5th year) were all under $20 \%$. The majority of the students were non-smokers (90\%); $5 \%$ were current smokers; and $5 \%$ were former smokers.

\section{Daily fat consumption of participating students}

Daily mean intake of total fat, saturated fat, and cholesterol based on students' characteristics is presented in Table 2. Results indicate that there was a significant difference in the consumption of total fat between male and female students. Daily mean intake of total fat was 
Table 2 Daily mean intake of dietary fats by students' characteristics

\begin{tabular}{|c|c|c|c|c|}
\hline & & Total fat (g) & Saturated fat (g) & Cholesterol (g) \\
\hline \multicolumn{5}{|l|}{ Variables } \\
\hline \multicolumn{5}{|l|}{ Gender } \\
\hline & Males & $85.9 \pm 24.8$ & $28.9 \pm 9.1$ & $298.2 \pm 79.3$ \\
\hline & Females & $92.0 \pm 18.8$ & $23.5 \pm 6.9$ & $224.5 \pm 61.7$ \\
\hline & $P$-value* & 0.0427 & $<.0001$ & $<.0001$ \\
\hline \multicolumn{5}{|c|}{ Body mass index } \\
\hline & $\mathrm{BMI}<18.5$ & $81 \pm 19.7$ & $22 \pm 8.3$ & $222.3 \pm 69$ \\
\hline & BMI 18.5- 24.9 & $91.6 \pm 20.8$ & $25 \pm 7.9$ & $242.1 \pm 74.6$ \\
\hline & $\mathrm{BMI}>25$ (overweight) & $85.3 \pm 19.3$ & $24.6 \pm 7.7$ & $248.1 \pm 73.9$ \\
\hline & BMI > 30 (obese) & $95.8 \pm 25$ & $29.2 \pm 8.7$ & $285.2 \pm 79.2$ \\
\hline & $P$-value** & 0.1120 & 0.1431 & 0.1414 \\
\hline \multicolumn{5}{|c|}{ Year in school } \\
\hline & 1st-year undergraduate & $90.9 \pm 24.2$ & $25.3 \pm 9.5$ & $250.4 \pm 89.5$ \\
\hline & 2nd -year undergraduate & $93.7 \pm 21.2$ & $25.9 \pm 7.8$ & $250.1 \pm 70.6$ \\
\hline & 3rd -year undergraduate & $87.2 \pm 18.2$ & $23.9 \pm 7.5$ & $236.7 \pm 75$ \\
\hline & 4th -year undergraduate & $91.8 \pm 20.1$ & $25.7 \pm 7.9$ & $249.6 \pm 73$ \\
\hline & 5th -year undergraduate & $87.1 \pm 23.1$ & $24.6 \pm 8$ & $242.8 \pm 74.1$ \\
\hline & $P$-value** & 0.4175 & 0.6716 & 0.8536 \\
\hline \multicolumn{5}{|c|}{ Major of study } \\
\hline & Science & $85.6 \pm 19.7$ & $22.8 \pm 6.9$ & $224.1 \pm 62.7$ \\
\hline & Non-Science & $95.1 \pm 21.4$ & $27.5 \pm 8.4$ & $268 \pm 80.5$ \\
\hline & $P$-value* & 0.0006 & $<.0001$ & $<.0001$ \\
\hline \multirow[t]{3}{*}{ Ethnicity } & White & $91 \pm 20.2$ & $25.2 \pm 7.8$ & $242.6 \pm 73.6$ \\
\hline & Black & $83.4 \pm 26.4$ & $23.7 \pm 9.4$ & $269.8 \pm 85.5$ \\
\hline & $P$-value* & 0.0996 & 0.3916 & 0.0996 \\
\hline \multicolumn{5}{|c|}{ Residential status } \\
\hline & On-Campus & $90.7 \pm 19.7$ & $25.3 \pm 7.6$ & $248.5 \pm 72.7$ \\
\hline & Off-Campus & $89.1 \pm 23.4$ & $24.5 \pm 8.7$ & $240.3 \pm 80$ \\
\hline & $P$-value ${ }^{*}$ & 0.5804 & 0.4669 & 0.4381 \\
\hline \multirow[t]{3}{*}{ Dieting } & No & $91.6 \pm 21$ & $25.6 \pm 8$ & $250.2 \pm 75.4$ \\
\hline & Yes & $83.8 \pm 19.5$ & $22.9 \pm 7.4$ & $226.7 \pm 70.8$ \\
\hline & $P$-value* & 0.0277 & 0.0478 & 0.0666 \\
\hline \multicolumn{5}{|c|}{ Smoking status } \\
\hline & Non-smoker & $90.2 \pm 20.5$ & $24.9 \pm 7.9$ & $243.2 \pm 74.4$ \\
\hline & Current smoker & $96.6 \pm 18.7$ & $29.3 \pm 7$ & $285.6 \pm 66.8$ \\
\hline & Former smoker & $83.2 \pm 29.3$ & $24.4 \pm 10.2$ & $249.3 \pm 88.8$ \\
\hline & $P$-value** & 0.2940 & 0.1427 & 0.1409 \\
\hline
\end{tabular}

* $T$-test for independent samples

**1-way ANOVA F-statistic

higher among females (92 g/day) compared to males $(85.9 \mathrm{~g} /$ day $)(P<0.001)$. However, saturated fat and cholesterol consumptions were lower for females (23.5 g/day) than males $(28.9 \mathrm{~g} /$ day $)$.

Regarding the year in school, students' daily mean intake of total fat did not significantly differ among students, ranging between $87.1 \mathrm{~g}$ (lowest) for the 5th year students and $93.7 \mathrm{~g}$ (highest) for 3rd year students. Likewise, mean saturated fat intake and cholesterol ranged between $23.9 \mathrm{~g}$ and $236.7 \mathrm{~g}$ (lowest) for the 3rd year students to $25.9 \mathrm{~g}$ and $250.1 \mathrm{~g}$ (highest) for the 2nd year students. There was also no significant difference 
between white and African American students in daily total mean intake of total fat, saturated fat, and cholesterol. Also, students' residential status and smoking habit were not associated with daily mean intake of total fat, saturated fat, and cholesterol.

On the other hand, students' daily mean values of total fat intake, saturated fat, and cholesterol were significantly different based on the major of study and dieting. Students majoring in science consumed lower amounts of total fat $(85.6 \mathrm{~g})$, saturated fat $(22.8 \mathrm{~g})$, and cholesterol (224.1 g) compared to non-science majors (95.1 g, $27.5 \mathrm{~g}$, and $268 \mathrm{~g}$, respectively) $(P<0.0001)$. Likewise, students who were on a diet consumed less total fat $(P<0.0277)$, saturated fat $(P<0.0478)$ and cholesterol compared to students who were not following any diet (Table 2). While the mean cholesterol intake was lower for students on a diet (225.7 g vs $250.2 \mathrm{~g})$, this difference did not achieve statistical significance $(P=0.0666)$.

\section{Nutrition knowledge survey}

Table 3 shows the results of the students' responses to the nutrition knowledge survey, indicating that female students scored higher than male students $(67.4 \pm 12.0$ vs. $62.5 \pm 15.7$, respectively, out of possible 105 points, $P=0.01$ ). Students who consumed less than $35 \%$ of daily calories from fat had a higher nutrition score $(P<0.0001)$ (Fig. 1$)$ and, similarly, students consuming less than $300 \mathrm{mg}$ of cholesterol had a higher nutrition score $(P=0.002)$ (Table 3$)$ (Fig. 2$)$.

Results of the students' responses to the four parts of the nutrition knowledge survey showed that students scored highest on the "Dietary Recommendations" section compared to the other two sections (Source of Foods/Nutrients and Diet-Disease Relationship). Female students scored higher than male students on all 3 parts of the nutrition knowledge survey, particularly on the "Dietary Recommendations" and the "Diet-Disease

Table 3 Mean scores of nutrition knowledge survey

\begin{tabular}{llll}
\hline & $n$ & Mean NK score & $P$-value \\
\hline $\begin{array}{l}\text { Total population } \\
\text { Gender }\end{array}$ & 231 & $66.0 \pm 13.4$ & \\
$\quad$ Male & 67 & $62.5 \pm 15.7$ & 0.01 \\
$\quad$ Female & 164 & $67.4 \pm 12.0$ & \\
$\begin{array}{l}\text { Percent fat } \\
<35 \%\end{array}$ & 139 & $69.8 \pm 13.0$ & $<.0001$ \\
$>35 \%$ & 92 & $61.2 \pm 12.4$ & \\
Cholesterol & & & 0.0002 \\
$<300 \mathrm{mg}$ & 181 & $67.7 \pm 12.7$ & \\
$>300 \mathrm{mg}$ & 50 & $59.8 \pm 13.9$ & \\
\hline
\end{tabular}

*T-Test for independent Samples
Relationships" sections, where scores were statistically different between genders (Table 4).

Controlling for gender, height, weight, age and dieting, there was a significant association between the students' intake of total fat, saturated fat, and cholesterol and nutrition knowledge score $(P<0.001)$ (Table 5).

\section{Discussion}

The 2010 Dietary Guidelines for Americans recommend that consumption of saturated fat should be limited to $<10 \%$ of daily calories, dietary cholesterol to $<300 \mathrm{mg}$ per day, and trans fat to $<1 \%$ of daily calories or as little as possible, primarily to reduce risk of CVD (9). This study looked at the association between nutrition knowledge and fat consumption in a sample of CMU students. Our findings support the general assumption that students' nutrition knowledge is associated with improved food choices pertaining to types of dietary fats, and the findings concur with results of previous studies [35-37].

In this study, nutrition knowledge was negatively correlated with fat and cholesterol intake. Students who consumed more than $35 \%$ of calories from fat or $>300 \mathrm{mg}$ of cholesterol daily had lower mean nutrition scores than those students with lower fat or cholesterol intake (8 points lower and 7.9 points lower, respectively) $(P<0.001)$. Using linear regression for nutrition scores on estimated saturated fat intake and cholesterol intake (controlling for gender, height, weight, age, and dieting), nutrition scores were negatively associated with saturated fat intake $(-0.15, P<0.0001)$ (Fig. 1$)$ and cholesterol intake $(-1.38, P<0.0001)$ (Fig. 2$)$. The results are correlational and therefore cannot show directionality. However, it is likely that nutrition education/ knowledge can lead to improvement of students' eating habits and healthier food choices. In general, to be able to understand and apply the Dietary Guidelines recommendations for healthful eating practices, some basic understanding of nutrition is necessary [36]. Mazier and McLeod reported that a single course in nutrition was effective at improving the nutrition knowledge of their undergraduate students [37]. A previous study conducted among 269 undergraduate Canadian students to examine the impact of nutrition education on fat consumption found that students who had taken a nutrition course consumed less fat than those with no nutrition education [38]. In this study, students with greater nutrition knowledge consumed lower amounts of total fat, saturated fat, and cholesterol per day compared with students with lower nutrition knowledge scores (Figs. 1 and 2). Proper nutrition knowledge is useful in improving dietary habits, and students are empowered when they have the necessary nutrition knowledge and skills needed to make healthful lifestyle choices [39-41]. 


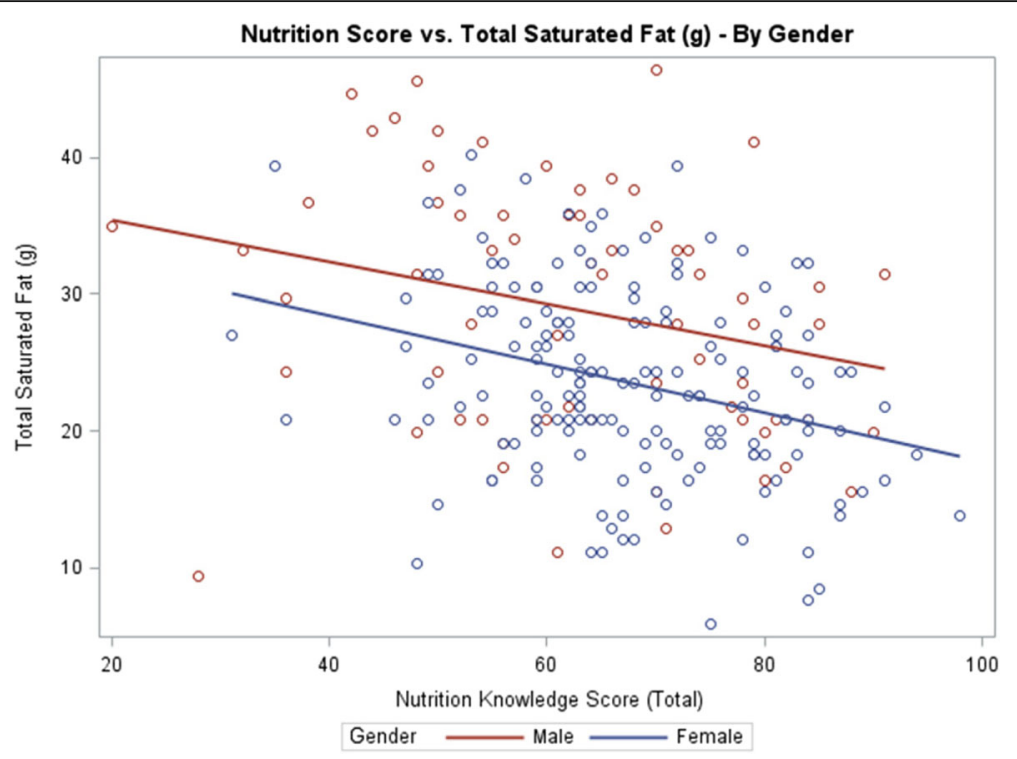

Fig. 1 Association of Nutrition Knowledge Score (Total) vs. Total Saturated Fat Intake by Gender

Regarding differences in gender, in this study, female students had greater nutrition knowledge than male students (the mean nutrition score for women was 5 points higher than that of men $(P=0.01))$. This finding was not surprising. In general, women are more likely than men to be interested in diet, nutrition, and body weight, particularly during college years [42-45]. A previous study conducted among 479 Swedish university students found that female students had healthier habits than male students, despite being stressed, whereas male students showed a high level of overweight and obesity and were less interested in nutrition advice and health-enhancing activities [44]. Also, the authors reported that female students were more interested in changing their dietary habits than male students were [44]. Another study evaluating the health knowledge of 428 African American university students found that $75 \%$ of students exhibited high levels of health knowledge, and female students displayed higher levels of knowledge than male students [45]. In our study, female students consumed less saturated fat and cholesterol than male students. This finding is consistent with a previous study among 184 Iranian female students aged 18-35 years showing that female students who perceived themselves as being in the healthy

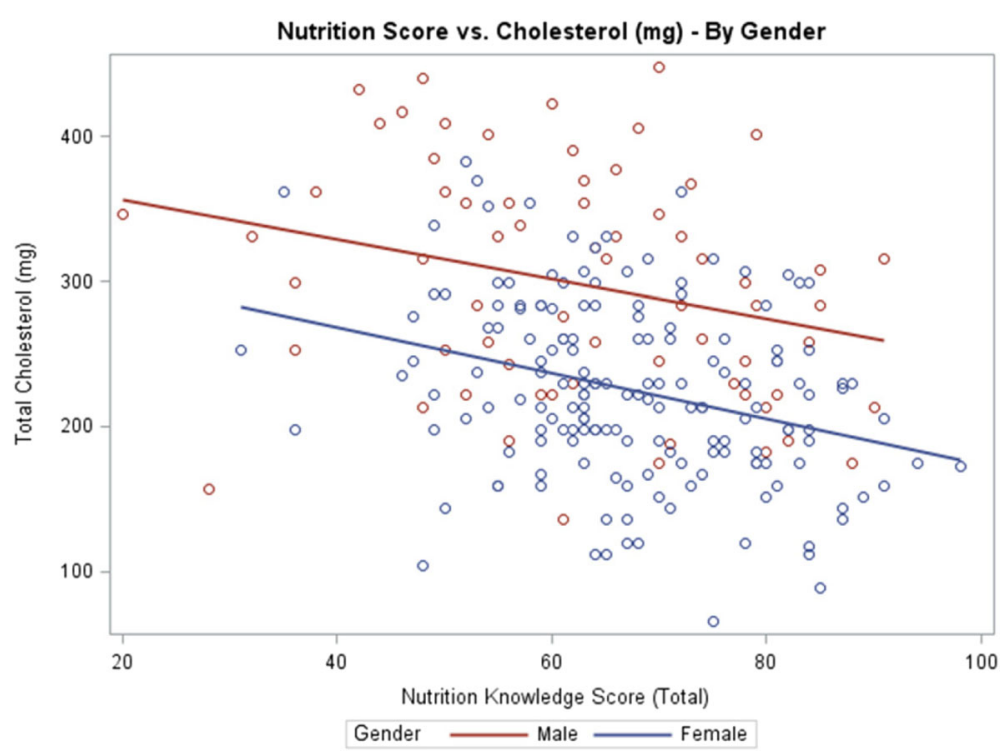

Fig. 2 Association of Nutrition Knowledge Score (Total) vs. Cholesterol Intake by Gender 
Table 4 Mean scores of nutrition knowledge survey and fat screening questionnaire by gender

\begin{tabular}{lllll}
\hline & Total score & Male & Female & $P$ value* \\
\hline Nutrition knowledge sections (total points) & & & & \\
Dietary recommendations (11) & $8.1 \pm 1.7$ & $7.6 \pm 2$ & $4.3 \pm 1.5$ & $44.1 \pm 8$ \\
Sources of foods/nutrients (69) & $43.4 \pm 8.6$ & $41.8 \pm 9.9$ & $5.6 \pm 1.8$ & 0.002 \\
Choosing everyday foods (10) & $5.5 \pm 1.8$ & $5.2 \pm 1.9$ & $9.4 \pm 3.5$ & 0.10 \\
Diet-disease relationships (15) & $8.9 \pm 3.8$ & $7.9 \pm 4.5$ & 0.01 \\
\hline
\end{tabular}

${ }^{*} T$-Test for independent Samples

weight range had a significantly lower intake of saturated fat and higher intake of monounsaturated fat compared to other students [46].

The results also showed that students' daily mean intake of total fat, saturated fat, and cholesterol were significantly different based on major of study and whether they were dieting. Students majoring in science consumed lower amounts of fat compared to nonscience majors $(P<0.0001)$. Likewise, students who were on a diet consumed less total fat, saturated fat, and cholesterol than students who were not following any diet $(P<0.0277)$. It is likely that students majoring in science would have covered more coursework related to nutrition than non-science majors. Also, students following a diet would be more likely to restrict their daily fat intake [42, 47]. Nevertheless, in this study, students' daily mean intake of total fat, saturated fat, and cholesterol did not differ by body mass index, year-inschool, ethnicity, residential living condition, or smoking status among students. These findings were not surprising [38, 48, 49]. Regarding fat intake, fat intake per se is not the only factor that affect body weight since a clustering of factors including heredity, a low level of physical activity, poor diet, and smoking can contribute to weight gain [50]. Likewise, students' nutrition knowledge will likely not increase without students taking a nutrition class, irrespective of how long students stay in college. Emrich and Mazier looked at the impact of nutrition knowledge on year-in-school and reported no significant differences in fat intake between first-year students and fourth-year students.

Table 5 Association between fat intake and nutrition knowledge score $^{a}$

\begin{tabular}{llll}
\hline & $\begin{array}{l}\text { Partial } \\
\text { correlation }^{\text {a }}\end{array}$ & $\begin{array}{l}\text { Regression } \\
\text { parameter }^{\mathrm{b}}\end{array}$ & $P$ value $^{* * *}$ \\
\hline Total fat intake $(\mathrm{g})$ & -0.27 & -0.42 & $<.0001$ \\
Saturated fat $(\mathrm{g})$ & -0.27 & -0.15 & $<.0001$ \\
Cholesterol $(\mathrm{mg})$ & -0.27 & -1.38 & $<.0001$ \\
\hline
\end{tabular}

Partial Correlation with Nutrition Score; controlling for gender, height, weight and age

${ }^{\mathrm{b}}$ Multiple linear regression of nutrition scores on Total Fat, saturated fat intake or cholesterol intake (controlling for gender, height, weight, age, and self-reported dieting)

***P-value for correlation and regression parameter
However, the authors reported that there were significant differences in fat intake between first-year science students with some nutrition education and those without [38]. This would suggest that nutrition education rather year-in-school is influencing students' fat intake.

In contrast to our results, which did not reveal any significant differences between on- and off-campus students, Emrich and Mazier reported differences in fat intake between students living on campus and off campus [38]. This could be due to the fact that the residential dining facility at CMU has a program called "Just $4 \mathrm{U}$ Nutrition", which offers students all types of healthy and balanced meal plans including vegetarian, vegan, Kosher, low-fat, cholesterol-free, and gluten-free meals. Students also have the option to choose an individualized meal plan that matches their lifestyle. However, our results were in agreement with a previous study conducted among 210 Iranian students to determine factors associated with nutrition knowledge and body weight, which found no significant correlation between nutritional knowledge, body mass index, and smoking status [49].

\section{Study's limitations}

This study is limited in its small sample size and in that most of the participating students were female $(71 \% \mathrm{fe}$ male vs. $29 \%$ male). It is possible that female students may have been more interested in research related to health issues than male students since students voluntarily entered into the study. Nevertheless, the dominance of female participants reflects the university's student body data and is consistent with the gender composition of previous studies [51, 52]. The limitations of any food frequency questionnaire (FFQ) and FFQ screeners (such as Block Fat Screener) are well recognized [53]. Although Block Fat Screener cannot estimate dietary fat intake accurately, the purpose of this study was not to estimate the amount of dietary fat intake in college students but rather to examine the associations between dietary fat intake level and nutrition knowledge, which is an appropriate use of the FFQ screener in research. Because a FFQ screener is composed of a pre-specified food list or set of behavioral questions, any single screener may not reflect the eating patterns of a given 
population. Therefore the generalizability of our findings to other populations is limited.

\section{Conclusion}

This pilot study found that students with more nutritional knowledge consumed less unhealthy fats and cholesterol. Students are empowered when they have the necessary knowledge and skills needed to make healthful lifestyle choices [54]. Given the importance of healthy eating in reducing CVD risk factors among students, future research on this topic is needed among this vulnerable age group. Factors such as food cost, food preparation, and cooking methods should also be included in future research since these factors can impact how effectively students can apply nutritional knowledge into their everyday eating habits. In conclusion, the study's results suggest that students were able to translate nutrition knowledge into their daily diet by reducing their saturated fat and cholesterol intake. This finding magnifies the role of nutrition education as a potential tool in health campaigns to promote healthy eating patterns among college students. We believe that the results of this pilot study can inform the design of future nutrition education intervention studies to assess the efficacy of nutrition knowledge on pattern of fat consumption among college students.

\section{Additional files}

Additional file 1: Consent Form. A sample of the consent form that was given to participants in this study. (TXT $5 \mathrm{~kb}$ )

Additional file 2: A copy of the study's questionnaire. (XML $412 \mathrm{~kb}$ )

Additional file 3: A copy of the raw data file. (CSV $157 \mathrm{~kb}$ )

Additional file 4: Copy of Regression and Correlation Analysis (CSV 996 bytes)

Additional file 5: SAS data analyses file. (DOCX 19 kb)

\section{Abbreviations}

BRFSS: Behavioral risk factor surveillance system; CDC: Centers for disease control and prevention; CVD: Cardiovascular disease

\section{Acknowledgments \\ A special note of appreciation goes to Dr. Allan Geliebter and Professor Steven Couture for reviewing the draft. Also, I would like to extend my sincere appreciation to all CMU students who participated in this study. In addition, a thank you note goes to Tanita Corporation for providing the body fat analyzer scale for this research.}

\section{Funding}

This work was partially supported by the Faculty Research and Creative Endeavors (FRCE) Premier Display grant at CMU.

\section{Availability of data and materials}

Data are available at cbrown03@bu.edu. Attached are copies of the statistical analysis.

\section{Authors' contributions}

NY carried out questionnaire design, manuscript preparation, data collection and study coordination. CB performed all the statistical analysis. MR contributed in data collection and data entry. MC reviewed all the statistical analysis. All authors read and approved the final manuscript.

\section{Competing interests}

The authors declare that they have no competing interests.

\section{Consent to publish}

Not applicable.

\section{Ethics approval and consent to participate}

Students agreeing to participate in the study were asked to sign a consent form, in harmony with the Helsinki declaration, and were informed that they could withdraw from the study at any time without penalty. The study protocol was approved by the Central Michigan University Institutional Review Board (IRB). IRB can be reached at: cmuirb@cmich.edu and the project ID is: IRBNet ID: 278950-2.

\section{Author details}

${ }^{1}$ Department of Human Environmental Studies, Central Michigan University, Wightman 108, Mt. Pleasant, MI 48859, USA. ${ }^{2}$ Jean Mayer USDA Human Nutrition Center on Aging at Tufts University, Boston, MA, USA. ${ }^{3}$ Department of Public Health and Family Medicine, Tufts University School of Medicine, 136 Harrison Avenue, Jaharis 264, Boston, MA 02111, USA. Department of Biostatistics, Boston University, 801 Massachusetts Avenue, Boston, MA 02118 USA.

Received: 1 April 2016 Accepted: 22 September 2016

Published online: 04 October 2016

\section{References}

1. Go AS, Mozaffarian D, Roger VL, Benjamin EJ, Berry JD, Blaha MJ, et al. American Heart Association Statistics Committee and Stroke Statistics Subcommittee. Heart disease and stroke statistics-2014 update: a report from the American Heart Association. Circulation. 2014;129:e28-e292. doi:10.1161/01.cir.0000441139.02102.80.

2. Murphy SL, Xu JQ, Kochanek KD. Deaths: Final data for 2010. Natl Vital Stat Rep. 2013;61(4):1-117.

3. CDC. Vital Signs: avoidable deaths from heart disease, stroke, and hypertensive disease-United States, 2001-2010. MMWR 2013; 62(35):721-7

4. Michigan Department of Community Health. February 2015 Michigan Health Statistics. Division for Vital Records and Health Statistics. Available at : https://www.michigan.gov/documents/mdch/CVH_fact_sheet_update-_ Final_3.4.15_483077_7.pdf

5. Centers for Disease Control and Prevention, National Center for Chronic Disease Prevention and Health Promotion, Division of Population Health. BRFSS Prevalence \& Trends Data [online]. 2015. [Accessed Oct 12, 2015]. URL: http://www.cdc.gov/brfss/brfssprevalence/.

6. Centers for Disease Control and Prevention. Chronic Diseases and Health Promotion. May 2014

7. Lichtenstein $\mathrm{AH}$, Appel $L$, Brands $\mathrm{M}$, et al. Diet and lifestyle recommendations revision 2006: a scientific statement from the American Heart Association Nutrition Committee. Circulation. 2006;114:82-96.

8. Mozaffarian D, Benjamin EJ, Go AS, Arnett DK, Blaha MJ, Cushman M, de Ferranti S, Després J-P, Fullerton HJ, Howard VJ, Huffman MD, Judd SE, Kissela BM, Lackland DT, Lichtman JH, Lisabeth LD, Liu S, Mackey RH, Matchar DB, McGuire DK, Mohler ER 3rd, Moy CS, Muntner P, Mussolino ME, Nasir K, Neumar RW, Nichol G, Palaniappan L, Pandey DK, Reeves MJ, Rodriguez CJ, Sorlie PD, Stein J, Towfighi A, Turan TN, Virani SS, Willey JZ, Woo D, Yeh RW, Turner MB; on behalf of the American Heart Association Statistics Committee and Stroke Statistics Subcommittee. Heart disease and stroke statistics-2015 update: a report from the American Heart Association [published online ahead of print December 17, 2014]. Circulation 2014. doi:10.1161/CIR.0000000000000152.

9. U.S. Department of Agriculture, U.S. Department of Health and Human Services. Dietary Guidelines for Americans, 2010. Washington, D.C: U.S. Government Printing Office; 2010.

10. Kotler LA, Cohen P, Davies M, Pine DS, Walsh BT. Longitudinal relationships between childhood, adolescent, and adult eating disorders. J Am Acad Child Adolesc Psychiatry. 2001;40:1434-40.

11. Neumark-Sztainer D, Wall M, Larson NI, Eisenberg ME, Loth K. Dieting and disordered eating behaviors from adolescence to young adulthood: findings from a 10-year longitudinal study. J Am Diet Assoc. 2011;111(7):1004-11. 
12. Nelson M, Story M, Larson N, Neumark-Sztainer D, Lytle L. Emerging adulthood and college-aged youth: An overlooked age for weight-related behavior change. Obesity. 2008;16:2205-11.

13. Lien N, Lytle LA, Klepp KI. Stability in consumption of fruit, vegetables, and sugary foods in a cohort from age 14 to age 21. Prev Med. 2001;33:217-26.

14. Racette SB, Deusinger SS, Strube MJ, Highstein GR, Deusinger RH. Weight changes, exercise, and dietary patterns during freshman and sophomore years of college. J Am Coll Health. 2005;53:245-51.

15. Sira N, Pawlak R. Prevalence of overweight and obesity, and dieting attitudes among Caucasian and African American college students in Eastern North Carolina: A cross-sectional survey. Nutr Res Pract. 2010;4(1):36-42.

16. Sulliman K, Rodas-Fortier K, Neyman M. A survey of dietary and exercise habits and perceived barriers to following a healthy lifestyle in a college population. Californian J Health Promot. 2004;2(2):10-9.

17. Anding JD, Suminski RR, Boss L. Dietary intake, body mass index, exercise, and alcohol: Are college women following the dietary guidelines for Americans? J Am Coll Health. 2001;49(4):167-71

18. Papadaki G, Hondros JA, Scott M. Kapsokefalou. Eating habits of university students living at, or away from home in Greece. Appetite. 2007:49(1):16976. doi:10.1016/j.appet.2007.01.008.

19. Deliens T, Clarys P, De Bourdeaudhuij I, Deforche B. Determinants of eating behaviour in university students. A qualitative study using focus group discussions. BMC Public Health. 2014;14(1):53. doi:10.1186/1471-2458-14-53.

20. Butler SM, Black DR, Blue CL, Gretebeck RJ. Change in diet, physical activity, and body weight in female college freshman. Am J Health Behav. 2004;28(1):24-32.

21. Chourdakis M, Tzellos T, Pourzitaki C, Toulis KA, Papazisis G, Kouvelas D. Evaluation of dietary habits and assessment of cardiovascular disease risk factors among Greek university students. Appetite. 2011;57(2):377-83.

22. The U.S. Surgeon General. National Prevention Strategy/Healthy Eating 2014. Available at http://www.surgeongeneral.gov/priorities/prevention/ strategy/healthy-eating.html. Accessed 25 Mar 2016.

23. U.S. Department of Health and Human Services: The Surgeon General's Vision for a Healthy and Fit Nation. Rockville, MD. U.S. Department of Health and Human Services, Office of the Surgeon General, 2010. [Available at http://www.surgeongeneral.gov/library/obesityvision/obesityvision2010.pdf. Accessed 25 Mar 2016

24. Ha EJ, Caine-Bish N. Effect of nutrition intervention using a general nutrition course for promoting fruit and vegetable consumption among college students. J Nutr Educ Behav. 2009;41(2):103-9.

25. Ha EJ, Caine-Bish N. Interactive introductory nutrition course focusing on disease prevention increased whole-grain consumption by college students. J Nutr Educ Behav. 2011;43(4):263-7.

26. Lin LP, Dali WP. The impact of nutrition education interventions on the dietary habits of college students in developed nations: a brief review. Malays J Med Sci. 2012;19(1):4-14

27. Brown ON, O'Connor LE, Savaiano D. Mobile MyPlate: a pilot study using text messaging to provide nutrition education and promote better dietary choices in college students. J Am Coll Health. 2014;62(5):320-7.

28. Shahril MR, Wan Dali WPE, Lua PL. A 10-Week Multimodal Nutrition Education Intervention Improves Dietary Intake among University Students: Cluster Randomised Controlled Trial. J Nutr Metab. 2013;2013:658642. https://www.hindawi.com/journals/jnme/2013/658642/.

29. Douglas PD, Douglas JG. Nutritional knowledge and food practices of high school athletes. J Am Diet Assoc. 1984;84:1198-202.

30. Perron DK, Endres J. Knowledge, attitudes and dietary practices of female athletes. J Am Diet Assoc. 1985;85:573-7.

31. Ozdogan $Y$, Ozcelik AO. Evaluation of the nutrition knowledge of sports department students of universities. J Int Soc Sports Nutr. 2011:8:11. doi:10.1186/1550-2783-8-11.

32. Clinical Guidelines on the Identification, Evaluation, and Treatment of Overweight and Obesity in Adults-The Evidence Report. National Institutes of Health. Obes Res. 1998;6 Suppl 2:51S-209S.

33. Block $G$, Gillespie $C$, Rosenbaum EH, Jenson C. A rapid food screener to assess fat and fruit and vegetable intake. Am J Prev Med. 2000;18(4):284-8. Available at: http://www.nutritionquest.com/wellness/free-assessment-toolsfor-individuals/fat-intake-screener/.

34. Parmenter K, Wardle J. Development of a Nutrition Knowledge Questionnaire. Eur J Clin Nutr. 1999:53:298-308.
35. Mitchell SJ. Changes after taking a college basic nutrition course. J Am Diet Assoc. 1990:90(7):955-61.

36. Worsley A. Nutrition knowledge and food consumption: can nutrition knowledge change food behaviour? Asia Pacific J Clin Nutr. 2002;11:S579-85.

37. Mazier MJ, McLeod SL. University science students' knowledge of fats. Can J Diet Pract Res. 2007;68(3):154-9.

38. Emrich TE, Mazier MJ. Impact of nutrition education on university students' fat consumption. Can J Diet Pract Res. 2009:70(4):187-92.

39. Contento P, Koch A, Lee H, Calabrese-Barton A. Adolescents demonstrate improvement in obesity risk behaviors after completion of choice, control \& change, a curriculum addressing personal agency and autonomous motivation. J Am Diet Assoc. 2010;110(12):1830-9.

40. Brown LB, Dresen RK, Eggett DL. College students can benefit by participating in a prepaid meal plan. J Am Diet Assoc. 2005;105:445-8

41. Wardle J, Haase AM, Steptoe A, Nillapun M, Jonwwutiwes K, Bellisle F. Gender differences in food choice: The contribution of health beliefs and dieting. Ann Behav Med. 2004;27(2):107-16.

42. Connor-Greene PA. Gender Differences in Body Weight Perception and Weight-Loss Strategies of College Students. Women Health. 1988;14(2):2742. doi:10.1300/J013v14n02_03.

43. Yahia N, El-Ghazale H, Achkar A, Rizk S. Dieting Practices and Body Image Perception among Lebanese University Students. Asia Pac J Clin Nutr. 2011;20(1):21-8.

44. von Bothmer MI, Fridlund B. Gender differences in health habits and in motivation for a healthy lifestyle among Swedish university students. Nurs Health Sci. 2005;7:107-18.

45. Livingston IL, Saafir BD, Manuel RC. Health knowledge among historically black college and university students: an exploratory study. Coll Student J. 2012;46(3):581-8.

46. Alipour B, Farhangi AM, Dehghan P, Alipour M. Body image perception and its association with body mass index and nutrient intakes among female college students aged 18-35 years from Tabriz, Iran. Eat Weight Disord Stud Anorexia Bulimia Obes. 2015;1:1-7.

47. Lowry R. Galuska, D A, Fulton, J E, Wechsler, H, Kann, L and Collins, J L. 2000. Physical activity, food choice, and weight management goals and practices among US college students. Am J Prev Med. 2000;18:18-27.

48. Wong JE, Parnell WR, Anna S, Howe AS, Lubransky AC, Katherine E, Black KE, Skidmore P. Diet quality is associated with measures of body fat in adolescents from Otago, New Zealand. Public Health Nutr. 2015;18:1453-60. doi:10.1017/S1368980014001645.

49. Zarei M, Taib MNM, Zarei F. Lifestyle factors and dietary intake of Iranian postgraduate students in Universiti Putra Malaysia (UPM). Electronic Physician. 2013:5(3):687-97. doi:10.14661/2013.687-697.

50. Arnaud C, Faeh D, Paccaud F, Cornuz J. Consequences of Smoking for Body Weight, Body Fat Distribution, and Insulin Resistance. Am J Clin Nutr. 2008;87(4):801-9.

51. Association ACH. American College Health Association-National College Health Assessment II: Reference Group Executive Summary Spring 2013. American College Health Association: Hanover, MD; 2013.

52. Yahia N, Brown C, Rapley M, Chung M. Assessment of college students' awareness and knowledge about conditions relevant to metabolic syndrome. Diabetology \& Metabolic Syndrome. 2014;6:111. doi:10.1186/ 1758-5996-6-111.

53. National Cancer Institute. National Institutes of Health. Dietary Assessment Primer, Screeners at a Glance; 2016 [Accessed on May 28, 2016]. Available from: https://dietassessmentprimer.cancer.gov/profiles/screeners/index.html

54. Taras H. Nutrition and student performance at school. J Sch Health. 2005; 75(6):199-213. 